\title{
CHANGING THE INFLUENCE OF PORTION SIZE ON CONSUMER BEHAVIOR VIA IMAGINED CONSUMPTION
}

\author{
Olivia Petit $^{1,2,}$, Charles Spence ${ }^{3}$, Carlos Velasco ${ }^{3,4}$, \\ Andy T. Woods ${ }^{3,5}$, \& Adrian D. Cheok ${ }^{1}$
}

Submitted September 2015

Revised June 2016

Accepted July 2016

${ }^{1}$ Imagineering Institute, Anchor 5, Mall of Medini, No. 4, Lebuh Medini Utara, IskandarPuteri, 79250, Malaysia.

${ }^{2}$ INSEEC Business School, 19 Quai de Bacalan, 33070, Bordeaux, France

${ }^{3}$ Crossmodal Research Laboratory, Department of Experimental Psychology, Tinbergen.

Building, 9 South Parks Road, Oxford, OX1 3UD England.

${ }^{4}$ Department of Marketing, BI Norwegian Business School, Nydalsveien 37, 0484 Oslo,

Norway

${ }^{5}$ Xperiment, Surrey, United Kingdom.

*Corresponding author: Olivia Petit, INSEEC Business School, 19 Quai de Bacalan, 33070,

Bordeaux, France. opetit@inseec.com, Tel: $+\underline{+33(0) 556007373}$ 


\begin{abstract}
A portion of food is usually considered as the norm for consumption. Due to the portion size effect, people tend to eat more when they are served a larger, as opposed to a smaller, portion. Here demonstrate that spontaneous simulations of the experience of eating a portion of food by consumers (what we calli.e., simulated eating) ean helped to reduce this portion size effect. Those participants who reported more eating simulations selected a smaller percentage of food from the very large portion. However, the quantity of food selected from this very large portion was nevertheless still larger than from the medium portion. Thus, simulated eating reduced but did not eliminate entirely the portion size effect. However, when the participants were encouraged to deliberatively imagine the sensory experiences associated with eating a portion of food (imagined eating), initial portion size no longer influenced the amount of food selected. Potential implications of these results for the consumer, for the food industry, and for public health are discussed.
\end{abstract}

Keywords: food consumption, social marketing, portion size, eating simulations, packaging, obesity. 


\section{CHANGING THE INFLUENCE OF PORTION SIZE ON CONSUMER BEHAVIOR VIA IMAGINED CONSUMPTION}

\section{Introduction}

Marketplace (individual) food portion sizes sold for immediate consumption have increased side-by-side with the rise in the prevalence of obesity in recent years. For example, it has been estimated that the portion sizes that are-available in supermarkets in the United States have increased 10-fold from 1970 to 2000 while the sizes of the glasses and bowls found in the home have increased by 36\% since 1960 (Wansink \& Van Ittersum, 2007). Crucially, portion size exerts a significant influence over energy intake. According to a recent meta-analysis of 211 studies, a doubling of portion size leads to an average $35 \%$ increase in the amount consumed (Zlatevska, Dubelaar, \& Holden, 2014). Importantly, the portion size effect does not appear to be affected by hunger (Rolls, Morris, \& Roe, 2002).

One explanation for the portion size effect is that the total content of a portion of food is perceived as the appropriate serving size (i.e., the appropriate amount to consume). Perhaps reflecting that, as children, we are often told by our parents to finish our plate, and are often rewarded for so doing (Birch, McPhee, Shoba, Steinberg, \& Krehbiel, 1987; Birch, Engell, \& Rolls, 2000; Versluis \& Papies, 2016). MoreoverFurthermore, given that people have trouble estimating the calorie content of a portion of food and hence their consequent energy intake (Rolls et al., 2002; Wansink \& Chandon, 2006), they tend to use the portion of food served as a benchmark against which to regulate their own consumption (Geier, Rozin, \& Doros 2006; Wansink, Painter, \& North, 2005). Thus, people tend to adjust their consumption behavior automatically as a function of the size of a food portion, which then leads them to eat more when presented with a larger portion than with a smaller portion (Rolls et al., 2002; Wansink 
et al., 2005).

According to Marchiori and Papies (2014, p. 41), facilitating mindful attention or "helping consumers to be aware of and rely on internal signals of hunger and satiety" might reduce the influence of the size of a food portion on consumption. In their study, the mindful intervention reduced the effects of hunger on unhealthy food consumption, but did not influence the portion size effect: That is, people continued to eat more from the larger food portion than from the smaller one. One explanation for the continued presence of the portion size effect was that the participants were only focusing on their internal hunger and satiety states, not on the eating experience that led up to these states (Petit et al., 2016a). It just so happens that such multisensory experiences - including the smell, taste, and mouth-feel of the food while eating - play an important role in the regulation of consumption (de Graaf, 2012; Ramaekers et al., 2014; Weijzen, Smeets, \& de Graaf, 2009). For example, the contact time of food in the mouth informs the brain of the likely inflow of nutrients, thus giving rise to satiety signals (de Graaf, 2012).

TIn this article, we explores whether the action of mentally simulating the experience of eating an entire portion (i.e., reenacting "perceptual, motor, and introspective states acquired during experience”, Barsalou, 2008, p. 618) might reduce the portion size effect. This, in turn, might benefit both consumers and also public health. For instance, if messages to encourage people to imagine the experience of eating the portion of food were added on a food's package, they might limit overconsumption from large food portions. Such messages might also increase people's desire to consume food in a smaller portion size. TIn the present research-we begin $\underline{\underline{s}}$ with a review of the relevant literature on portion size and mental simulation, followed by the outlining of our the hypotheses. Next, we present those studies that have been conducted in order to test these hypotheses are presented. Finally, we the article is concluded by addressing the specific contributions of the studies is addressed, as 
well as bynd presenting limits and directions for future research presented.

\section{Theory and Hypotheses}

It may appear paradoxical The fact that imagining the experience of eating an appetizing portion of food can both enhance the expected pleasure, and increase purchase intent while, at the same time, reducing food intake may appear paradoxical. For this reason, we examine the links between mental simulation and portion size and their effect on food consumption are examined.

\subsection{Food portion and sensory pleasure}

When deciding on a particular portion size, a consumer's strategy is partly guided by the expected pleasure associated with the food, as well as by the need to ensure that the size of the portion is large enough to stave-off hunger (Brunstrom \& Shakeshaft, 2009). One prediction, then, is that consumers should choose a larger portion of food when they are feeling hungry, and a smaller portion when they are not so hungry. However, consumers are often offered price discounts with larger portion sizes when they go shopping at the supermarket, or when eating at a restaurant, and this generally increases consumption (Chandon \& Wansink, 2012; Vermeer, Alting, Steenhuis, \& Seidell, 2010).

Logically, when presented with a large portion when not hungry, the consumer should just eat less of the larger portion. However, consumers automatically tend to eat more from larger portions than from smaller ones. Paradoxically, it would appear that the lack of enjoyment, during the consumption of the food, could perhaps help to explain the importance of the portion size effect. From a homeostatic point of view, people eat more when they feeling hungry in order to compensate for the decline of energy resources (Redden \& Haws, 2013). Oftentimes, they will stop eating once they have replenished these resources. Satiation is an indicator of this replenishment, which results in the decline in enjoyment of the food with greater consumption (de Graaf, 2012; Herman \& Polivy, 1983; Redden \& Haws, 2013). 
For this reason, hunger should lead people to cease eating once they feel less enjoyment (Herman \& Polivy, 1983). However, the size of the food portion suggests how much it is acceptable to eat (Wansink, 2004). The portion size effect seems to occur implicitly, since consumers eat more from a large portion even if they do not feel hungry (Kral, Roe, \& Rolls, 2004; Rolls et al., 2002), or the food itself is stale (Wansink \& Kim, 2005). Therefore, hunger and pleasure do not seem to play a primary role in the regulation of consumption when people eat their food in a portion format.

\subsection{Mental simulation and sensory pleasure}

According to the theory of grounded cognition, the our initial perception of an object is stored in memory and when the consumer later experience the object again, we they mentally simulate the prior perceptual experiences associated with that object (Barsalou, 2008; Barsalou, Simmons, Barbey, \& Wilson, 2003). Mental simulation can be conceptualized as a more automatic form of mental imagery initiated by the exposure to representations of objects (Elder \& Krishna, 2012). When people see a picture of a food product on an advertisement, they will simulate behavioral scenarios of using or eating the food product. This simulation may, in turn, influence, for example, their purchase intentions (Elder \& Krishna, 2012; Krishna \& Schwarz, 2014).

In the context of eating behavior, seeing attractive food appears to trigger spontaneous reenactments of previous encounters. These reenactments include thinking about the sensory (e.g., taste, texture, and temperature), situational (e.g., time, place, event), and hedonic (i.e., pleasure, displeasure) features of the food, something that is called "eating simulations" (Papies, 2013). People can also deliberatively attempt to construct representations of eating experiences in working memory that are labeled as "eating imagery" (Barsalou, 2008; Elder \& Krishna, 2012; Morewedge, Huh, \& Vosgerau, 2010).

In general, mental simulation has a motivational function in that it can facilitate 
interactions with the object, help prepare action, and, in the case of food consumption, may also increase food craving (Anderson, 1983; Gregory, Cialdini, \& Carpenter, 1982; Jeannerod, 2001; Schlosser, 2003; Spence, Okajima, Cheok, Petit, \& Michel, in press). For instance, eating simulations can be evoked by hunger and the energy content of food. These variables produce increased activity in those brain areas that are involved in the processing of taste and reward (i.e., the bilateral posterior fusiform gyrus, the left lateral orbitofrontal cortex, and the left middle insula) when people see pictures of food (Van der Laan, De Ridder, Viergever, \& Smeets, 2001). Thus, the extent of eating simulations might well be increased when consumers feel that afood is appetizing, hence increasing food craving (Papies, 2013; Spence et al., in press). Such a notion would be consistent with Elder and Krishna's (2012) finding that for those consumers who report more mental simulations of interactions with an appetizing food product, purchase intent increased. However, people do not necessarily increase their food intake as a consequence of the desire to acquire the food. Next, we discuss the positive effect of mental simulations on both the motivation to acquire the food and the regulation of consumption is discussed.

\subsection{Portion size and mental simulation}

Mental simulation can fuel sensory pleasure and may also give rise to satiation (Larson, Redden, \& Elder, 2014). Satiation is not solely driven by an internal signal indicating that physiological limits have been reached. Rather, there is also a top-down cognitive component to satiation has-also has a top-down cognitive component that is likely linked to sensory adaptation and habituation (Galak, Redden, \& Kruger, 2009; Redden, \& Haws, 2013). Mental simulation is at least partially represented in the same brain systems as overt perceptual states. Thus, eating simulations can reactivate some of the same brain areas that are recruited during actual consumption, which in turn, can produce similar sensations (Barsalou, 2008; Chen, Papies, \& Barsalou, in press; Simmons, Martin, \& Barsalou, 2005). 
Similar to consumption, then, the mental simulation of eating a large quantity of food may affect satiety and give rise to feelings of fullness (Larson et al., 2014; Toepel et al., 2015).

Larson et al. (2014) demonstrated that simply viewing 60 (vs. 20) pictures of food having a specific taste (e.g., a salty food) decreased people's enjoyment of similar tasting food during subsequent consumption. Larson and his colleagues provided evidence in support of the idea that the level of mental simulation of the taste of a pictured food mediates the effect of the picture on the food's enjoyment. At the neural level, viewing a portion that is "too big" is associated with less activity in those brain areas (e.g., the inferior parietal lobule, superior temporal gyrus, and mid-posterior cingulate gyrus), that are associated with attention and reward valuation compared to the sight of the "ideal" portion (Toepel et al., 2015). Mental imagery can also be used to encourage the consumer to make a healthy food choice and reduce their consumption (Morewedge et al., 2010; Petit et al., 2016b). For instance, asking a participant to imagine eating $30 \mathrm{M} \& \mathrm{Ms}$ (vs. just three) significantly reduced subsequent consumption of these candies in the lab setting (Morewedge et al., 2010).

However, if mental imagery can reduce consumption, it does not necessarily decrease the desire for the product imagined. First, when people imagine the consumption of a large quantity of food, they desire to eat less. Yet, that does not necessarily affect how much they like the food (Morewedge et al., 2010). Second, focusing people's attention on the sensory experience of eating the food appears to enhance the food's enjoyment and to increase their willingness to pay for smaller food portions (Cornil \& Chandon, in press). In the latter study, participants had to imagine the sensory features of three high-calorie foods (e.g., the crunching sound made by cereals when eaten, the oral-somatosensory sensation of chocolate melting in the mouth, the smell of waffles, etc.), prior to choosing a portion of another highcalorie food. Using multisensory imagery to imagine eating the food led people to choose smaller portions, to expect more enjoyment from eating, and to be willing to pay more for

Commented [CS9]:

Commented [CS10]:

Commented [CS11]:

Commented [CS12]:

Commented [CS13]:

Commented [CS14]: Avoid so many repetitions of same word? 
their smaller portion as well. For Cornil and Chandon, imagining the sensory experience associated with eating made people evaluate portions based on the expected sensory pleasure, which led the latter to choose smaller portions because they give rise to reduced sensory satiation (Garbinsky, Morewedge, \& Shiv, 2014). Consistent with this view, Brunstrom and Shakeshaft (2009) demonstrated that people tend to select smaller portions of those foods that they like and for which they expect more satiety (i.e., more reward) than food that is considered to be less rewarding.

As mental simulation has a motivational purpose, imagining the experience of eating food may increase the desire to obtain the food (Jeannerod, 2001; Schlosser, 2003).

Furthermore, simulating this experience in relation to a large amount of food can reduce hunger and food consumption (Larson et al., 2014; Morewedge et al., 2010), without necessarily decreasing the desire for the food (Cornil \& Chandon, in press). Thus, eurthe prediction is that those people who simulate more (vs. less) experiences of eating the portion of food will expect more pleasure (Hypothesis 1) and will exhibit higher purchase intentions (Hypothesis 2) regardless of the portion size. This, in turn, would suggest a higher (vs. lower) desire to obtain the food (Cornil \& Chandon, in press; Jeannerod, 2001; Schlosser, 2003, Fig. 1). However, since the size of the portion should affect satiety (Larson et al., 2014;

Morewedge et al., 2010), we suppose an interactive effect of the eating simulation and the food portion size on hunger is supposed (Hypothesis 3). More specifically, the expectation is that people who experience more (vs. less) eating simulations would feel less (vs. more) hungry after seeing the larger (vs. smaller) food portion. Finally, eur the main hypothesis is that those who experience more eating simulations should also be less affected by the portion size effect (Fig. 1). Thus, we expect an interactive effect of the eating simulation and the portion size on the amount of food selected (Hypothesis 4) is expected. People who simulate more (vs. less) experiences of eating the food portion would select less (vs. more) food from

Commented [CS15]: Postulated? Suggested?

Sopposed sounds odd 
the larger (vs. smaller) portion.

Fig. 1 here

\section{Study 1}

Zlatevska et al. (2014) recently demonstrated that people eat more when served a medium as compared to a small portion of food, and when served a large as compared to a medium-sized one. Interestingly, this effect became smaller for successively larger portionsize comparisons. This curvilinear relationship can be explained by the fact that quantity becomes more salient to the viewer when the portion size is very large because this portion is less likely to be considered as a consumption norm compared to smaller food portions. It is te be expected that This increase in the size of the food portion will-would be more salient for those individuals who simulate more experiences of eating the portion of food.

\subsection{Materials and methods}

142 participants (64 females, mean age $=29.80, S D=8.97$ ) took part in the online study, conducted using the HTML/JavaScript version of the Xperiment research package (www.xperiment.mobi; for considerations regarding online research see Woods, Velasco, Levitan, Wan, \& Spence, 2015). All of the participants were recruited using Prolific Academic (http://prolificacademic.co.uk/) in exchange for a payment of $£ 0.40$. They evaluated a portion of French fries in one of three experimental conditions: medium (45 participants), large (50 participants), and very large portions (47 participants), in a betweenparticipansubjects experimental design. Note that the different portions corresponded to three different portion sizes that can be found in McDonald's restaurants currently (http://www.mcdonalds.com/us/en/home.html). The medium portion size consisted of 17 French fries (i.e., $114 \mathrm{~g}$ of French fries or $340 \mathrm{kcal}$ ) presented in a medium package, the large portion contained 26 French fries (i.e., $175 \mathrm{~g}$ of French fries or $520 \mathrm{kcal}$ ), while the very large 
portion consisted of 51 French fries (i.e., $340 \mathrm{~g}$ of French fries or $1020 \mathrm{kcal}$ ) shown in a very large serving (see Appendix A.1).

After viewing the manipulated graphics of the French fries, the participants indicated "How much of the portion of French fries would you like to eat?" on a scale from "none" to "all of the portion". The scale was illustrated with the package of French fries occupying a fixed position on the screen, and the number of French Fries in the package being manipulated by the participant in order to reflect how many fries that they would like to eat (Appendix A.2). To estimate how accurately the participants perceived the size of the food portion, they were asked to estimate the caloric content of the French fries shown by answering the following question: "How many calories does the portion of French fries contain?" on a visual analogue scale from 0 to 1000 calories. The participants were asked about their hunger level, the appeal of the food, and their purchase intentions with 7-point scales via the following questions "How hungry are you at present?"; "How appetizing do you find the portion of French fries?"; "How likely would you be to buy this portion of French fries?" To measure the simulation of eating experiences, a three-item scale was used (Elder \& Krishna, 2012). The participants rated the extent to which mental images of eating the portion of French fries came to mind $(1=$ not at all; $7=$ very much so $)$, the number of images of eating the French fries that came to mind $(1=$ few or no images; $7=$ many mental images $)$, and the extent to which they could imagine eating the French fries $(1=$ not at all; $7=$ to a great extent). TWe combined these measures are combined to form a scale for the "number" of eating simulations $(\alpha=.88)$, indicating that the participants can report "more" or "less" mental simulation of their eating experiences.

\subsection{Results}

We decided to use $\underline{T}$ the median-split was used on our the continuous variable of simulation of eating experiences, because we were interested inin order to examininge group 
differences, and oursince the two independent variables (i.e., eating simulation and food portion size) were uncorrelated $(r=.046, \mathrm{n}=142, p=.587$, see Iacobucci, Posavac, Kardes, Schneider, \& Popovich, 2015). Thus, we had two groups of 71 participants- have beenwere

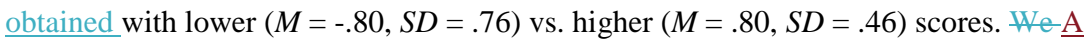
eonducted 2 × 3 analyses of variance (ANOVAs) were-was conducted with simulation of eating (lower scores, higher scores) and portion size (medium, large, very large) as the independent variables on eur the dependent variables. The descriptive statistics are summarized in Table 1. First, there was a signifieantthe main effect of mental imagery on the perceived calorie content was significant $\left(F(1,136)=10, p=.002, \eta^{2}\right.$ partial $\left.=.068\right)$. An examination of the means revealed that the participants with higher eating simulation scores perceived the portions of French fries as being more calorific $(M=31.80$ calories, $S D=$ $18.65)$, than those with lower scores $(M=23.24, S D=12.70)$. Neither the main effect of portion size $(p=.107)$, nor the interaction between the two factors $(p$ 's $>.5)$ was significant.

Table. 1 here

Supporting Hypothesis 1, there was a significantthe main effect of simulated eating experiences on how appetizing the food looked was significant, $(F(1,136)=50.84, p<.001$, $\eta^{2}$ partial $=.272$ ). The participants with higher eating simulation scores evaluated the portion of French fries as more appetizing $(M=5.68, S D=1.24)$ than those with lower scores $(M=$ $4.01, S D=1.55)$. Neither the main effect of portion size $(p=.146)$, nor the interaction between the two factors ( $p$ 's $>.5$ ), was significant.

Supporting Hypothesis 2, the main effect of simulated eating experiences on purchase intent was also significant $\left(F(1,136)=28.29, p<.001, \eta^{2}\right.$ partial $\left.=.172\right)$. Those participants with higher eating simulation scores reported greater purchase intent $(M=5.01, S D=1.62)$ than those with lower scores $(M=3.50, S D=1.80)$. Note that neither the main effect of portion size $(p=.105)$, nor the interaction between the two factors $(p$ 's $>.5)$, was 
significant.

The main effects of simulated eating experiences $F(1,136)=10.37, p=.002, \eta^{2}$ partial $=$ $.071)$ and of portion size $\left(F(2,136)=5.93, p=.003, \eta^{2}\right.$ partial $\left.=.080\right)$ on hunger were significant. Those participants with higher eating simulation scores reported being hungrier $(M=4.17, S D=1.69)$ than those with lower scores $(M=3.37, S D=1.64)$. The participants indicated that they were less hungry after seeing the very large portion $(M=3.15, S D=1.62)$ than after seeing the large $(M=4.00, S D=1.67)$ and medium portions $(M=4.16, S D=1.71)$. However, the interaction between mental simulation and portion size was not significant $(p=$ .172). Hypothesis 3 was therefore unot supported.

Our The key hypothesis (Hypothesis 4) regarded the interaction between portion size and the simulated eating of the amount of food selected in the portions. Because hunger was nonlinearly affected by portion size and decreased with the very large portion for all of the participants, it was deemed important to test that the effect of simulations does not result from changes in perceived hunger. For this reason, we decided to include hunger-was included as a covariate. We found that The main effect of portion size was significant after controlling for hunger $\left(F(2,135)=26.07, p<.001, \eta^{2}\right.$ partial $\left.=.279\right)$. An examination of the means revealed that people selected more French fries from the very large portion $(M=23.49$ French fries, $S D=14.00)$ than from either the large $(M=13.50$ French fries, $S D=8.50)$, or medium portions $(M=8.33$ French fries, $S D=6.90)$. Neither the main effect of simulation of eating experiences $(p=.287)$, nor the main effect of hunger $(p$ 's $>.5)$, was significant. Importantly, the interaction between the two factors reached significance $(F(2,135)=6.49, p$ $=.002, \eta^{2}$ partial $\left.=.088\right)^{1}$. The means are presented in Figure 2.

Simple effects tests revealed that the number of French fries selected in the medium

\footnotetext{
${ }^{1}$ The predicted interaction was also significant when the eating simulation variable was used as a continuous variable in a regression analysis $F(2,139)=25.129, p<.0001$.
} 
portion $(p=.168)$ and in the large portion $(p$ 's $>.5)$ were not significantly different between the two groups. However, as expected those participants with higher eating simulation scores selected less French fries $(M=18.60$ French fries, $S D=13.86)$ than those with lower scores $\left(M=29.06\right.$ French fries, $S D=12.21, F(1,45)=7.42, p=.009, \eta^{2}$ partial $\left.=.142\right)$, when presented with the very large portion.

There was a significant The linear tend, $(F(1,69)=8.16, p=.006, r=.33)$, indicating that as the portion size increased, the amount of food selected by people with higher eating simulation scores increased proportionally, was significant. Planned contrasts revealed that those participants selected significantly more French fries in the very large portion $(M=$ 18.60 French fries, $S D=13.86)$ than in the medium portion $(M=9.86$ French fries, $S D=$ 7.27, $t(69)=2.86, p=.006$, Cohen's $d=.77$ the other comparisons were not significant). However, as expected, there was a significantthe linear trend, $(F(1,69)=4.61, p=$ $.035, r=.25)$, indicating that as the portion increased, the percentage of food selected by those participants with higher eating simulation scores decreased proportionally, was significant. Planned contrasts revealed that those participants selected a significantly lower percentage of French fries in the very large $(M=36.47 \%, S D=27.18)$ than in the medium portion $(M=57.98 \%, S D=42.76, t(69)=2.15, p=.035$, Cohen's $d=.61)$. By contrast, the percentage of French fries selected by participants with lower eating simulation scores was not significantly different between the different portion sizes $(p=.197)$.

Fig. 2 here

\subsection{Discussion}

The results of Study 1 are largely supportive of Hypotheses 1 and 2. Participants with higher eating simulation scores reported finding the food more appetizing (H1), and expressed greater purchase intent (H2) after seeing the portion of French fries than those participants with lower scores. That said, we did not find the expected interaction between the 
simulation of eating and the food portion size on hunger levels was not foundobtained (H3). In particular, we expected that participants with higher (vs. lower) eating simulation scores would feel less (vs. more) hungry after seeing the larger (vs. smaller) food portions.

Otherwise, those participants with higher eating simulation scores reported being hungrier than those participants with lower scores in all of the conditions. A possible explanation is that people with higher eating simulation scores are more motivated to obtain the food (Jeannerod, 2001; Schlosser, 2003) and for this reason, indicated that they were hungrier, regardless of the portion size.

Concerning the effect of our the independent variables on food selection, the expected curvilinear relationship between portion size and the amount of food selected inside the portion for the participants with lower eating simulation scores was not found (as documented by Zlatevska et al., 2014). Participants did not select a different percent of fries as a function of the size of the food portion, although they did indicate that they were less hungry after seeing the very large portion. One interpretation for this result is that the participants in our the study were only asked to select the food that they would eat. That is, we did not ask them-they were not asked to actually consume the portion that they selected. Since people with lower eating simulation scores are less motivated to eat the food (less pleasure expectation and lower purchase intentions), it can be supposed that they would have been less affected by the increase of the portion size in their food selection.

However, eur the results suggest that the spontaneous simulation of eating experiences reduce the portion size effect. As hypothesized (H4), those participants with higher eating simulation scores selected less food than those participants with lower scores when presented with the very large portion. MoreoverIn addition, those participants selected a lower percentage of French fries from the very large portion than from the medium portion. However, they selected more French fries from the very large than from the smaller portion. 
As a result, the portion size effect was reduced in those people-participants with higher eating simulation scores, but it was not eliminated entirely. Following on from this, the second study was designed to tested whether by explicitly asking participants to imagine the sensory experience of eating the entire portion of food, the portion size effect can be diminished or indeed prevented entirely.

\section{Study 2}

It is also possible to prompt-Ppeople can also be prompted to explicitly imagine the consumption of food, for example, by deliberately asking them to imagine eating (Cornil \& Chandon, in press; Morewedge et al., 2010). People who repeatedly imagine eating a particular food many times over consume less of the imagined food subsequently (Morewedge et al., 2010). Similar to the first study, hypothesized-a significant interaction between the size of the food portion, and in this case, eating imagery on both the hunger (Hypothesis 3), and the number of foods selected was hypothesized (Hypothesis 4).

Spontaneous simulations of eating experience might reflect a greater motivation to obtain the food (Jeannerod, 2001; Schlosser, 2003), a finding that was confirmed by eur the results. However, in contrast with the first study, here, we explicitly asked-the participants were explicitly asked to imagine the sensory experience of eating. Therefore, there was no reason to expect that those individuals explicitly asked to imagine the food as being more motivated to obtain food, relative to those individuals who received no such instruction. Elder and Krishna (2012) demonstrated a significant interaction between the valence of the product and the participants' embodied simulation of the product on purchase intentions. They found that viewing advertising for a product that facilitates more (vs. less) mental simulations results in higher purchase intentions when this product is evaluated positively. By contrast, higher (vs. lower) simulations resulted in lower purchase intentions, when the product was 
evaluated negatively. For this reason, reformulated-Hypotheses 1 and 2 were

reformulated in this second study. In particular, predicted a significant interaction between the size of the food portion and mental imagery on expected pleasure and purchase intentions was predicted. The expectation was that people in the eating imagery (vs. control imagery) condition would find the food more (vs. less) appetizing and would have more (vs. less) purchase intentions, from the food portion size which is evaluated more (vs. less) positively.

\subsection{Material and methods}

A sample of 197 participants ( 80 females, mean age $=28.90, S D=9.20$ ) took part in the online study, conducted with Xperiment. All of the participants were recruited using Prolific Academic in exchange for payment of $£ 0.40$. The participants had to evaluate a chocolate bar in a 2 (food configuration: smaller vs. larger portion) x 2 (eating imagery vs. control imagery) between-subjects experimental design. The smaller food portion condition consisted of a picture of a 15 square chocolate bar (approximately $75 \mathrm{~g}$ and 400 calories), while the larger food portion included a picture of a 24 square chocolate bar (approximately $100 \mathrm{~g}$ and 530 calories, see Appendix B).

The 98 participants assigned to the eating imagery condition were instructed to imagine the sensory experience of eating all of the chocolate while looking at the picture. In the control imagery condition, the 99 participants were encouraged to "think about anything (they) you would like to think about". After viewing the manipulated graphics of the chocolate bar, the participants indicated "How many squares of chocolate you would like to eat?" on a scale from "0" to "all" of the portion. The scale was illustrated with a chocolate bar on the screen, with the number of chocolate squares varying as a function of the participant's answer (the participant used the cursor keys to change the number of pieces of visible chocolate). The other dependent variables included perceptions of caloric content, 
how appetizing the food looked, purchase intentions and eating simulations (see Study 1).

\subsection{Results}

For each dependent variable, separate 2 × 2 ANOVAs were conducted with the mental imagery condition and the portion size as independent variables. The descriptive statistics are summarized in Table 2. Checking the mental imagery manipulation, the amount of actual mental imagery reported by participants was found to be higher for those in the eating imagery condition $(M=5.15, S D=1.42)$ than for those in the control condition $(M=4.43$, $S D=1.79, F(1,193)=9.55, p=.002, \eta^{2}$ partial $\left.=.047\right)$. The amount of mental imagery was not affected by portion size $(p>.5)$, nor by the interaction of the imagery manipulation and portion size $(p=.084)$.

Table. 2 here

In terms of the dependent variable of calorie content, there was a significantthe main effect of mental imagery was significant $\left(F(1,193)=3.85, p=.051, \eta^{2}\right.$ partial $\left.=.020\right)$. The main effect of portion size was not significant $(p=.113)$, but the interaction between the two factors was significant $\left(F(1,193)=5.06, p=.026, \eta^{2}\right.$ partial $\left.=.026\right)$. A simple effects test revealed that those participants in the eating imagery condition rated the smaller chocolate bar $(M=37.10$ calories, $S D=15.59)$ as being more calorific than the larger bar $(M=27.51$, $S D=17.48, F(1,96)=8.24, p=.005, \eta^{2}$ partial $\left.=.079\right)$, whereas the calorie perception between the two portion sizes was not significantly different for those participants in the control imagery condition $(p$ 's $>.5)$. Those participants also estimated the chocolate bar to be more calorific than those in the control imagery condition when the smaller chocolate bar was displayed (vs. $M=26.59$ calories, $S D=17.52, F(1,97)=9.97, p=.002, \eta_{\text {partial }}^{2}=.093$ ). However, the perception of the calorie content was not significantly different between the two groups for the larger portion of food ( $p$ 's $>.5)$.

Supporting Hypothesis 1, there was a significantthe interaction between the mental 
imagery condition, and the size of the chocolate bar, on participants' perception of how appetizing the chocolate bar looked was significant $\left(F(1,193)=4.32, p=.039, \eta^{2}\right.$ partial $\left.=.022\right)$. Neither the main effect of mental imagery $(p=.157)$, nor the main effect of portion size was significant $(p$ 's $>.5)$. A simple effects test revealed that participants in the eating imagery condition rated the smaller chocolate bar as being more appetizing $(M=5.60, S D=1.44)$ than those in the control imagery condition $(M=4.78, S D=1.71, F(1,97)=6.72, p=.011$, $\eta^{2}$ partial $\left.=.065\right)$. However, those participants in the eating imagery condition did not find the larger chocolate bar to be any more appetizing than those participants in the control condition $(p$ 's $>.5)$. We did not find- $\underline{D}$ differences in expected pleasure between the two chocolate bars were not found, either for those participants in the control imagery condition ( $p=.157)$, or for those participants in the eating imagery condition ( $p$ 's $>.5$ ). MoreoverIn addition, we did not find-significant effects of the independent variables on purchase intentions and the level of hunger were not found. Thus, eur the results do not support Hypotheses 2 and 3.

Our The key hypothesis (H4) focused on the interaction effect between the portion size of the bar and the mental imagery condition on the amount of food selected. There was a signifieant The main effect of the-portion size was significant $(F(1,193)=10.24, p=.002$, $\eta^{2}$ partial $\left.=.050\right)$. Importantly, the interaction between the two factors was also significant $(F(1$, $193)=5.17, p=.024, \eta^{2}$ partial $\left.=.026\right)$. Fig. 3 graphically presents the means. A simple test of effects showed that participants in the control condition (no eating-imagery) selected significantly more chocolate squares in the larger portion $(M=11.60$ chocolate squares (i.e., $48.33 \%), S D=6.87)$ than in the smaller portion $(M=7.06$ chocolate squares (i.e., $47.08 \%)$, $S D=4.71, F(1,97)=14.64, p<.001, \eta^{2}$ partial $\left.=.131\right)$. Another simple test of effects revealed that for those participants, the percent of food selected in the smaller and larger food portions did not differ significantly ( $p$ 's $>.5$ ). By contrast, as expected, simple tests of effects highlighted that those participants in the eating imagery condition did not select significantly 
more chocolate in the larger portion than in the smaller food portion $(p$ 's $>.5)$. Those participants selected a lower percentage of the food from the larger portion $(M=40.57 \%$ (i.e., 9.69 chocolate's squares) $S D=26.74)$, than from the smaller portion $(M=59.47 \%$ (i.e., 8.92 chocolate's squares) $S D=33.07, F(1,96)=9.63, p=.003, \eta^{2}$ partial $\left.=.091\right)$.

Fig. 3 here

\subsection{Discussion}

The results of Study 2 are largely supportive of eur the main hypothesis (Hypothesis 4). Specifically, the interaction between the portion size and mental imagery on the amount of food selected was significant. As expected, asking the participants to imagine the sensory experience of eating the entire food portion, cancelled the portion size effect (Geier et al., 2006; Rolls et al., 2002; Wansink et al., 2005). Contrary to those participants in the control imagery condition who selected more chocolate squares in the larger than in the smaller food portion, the difference between the number of chocolate squares selected in the smaller portion and in the larger portion was not significant, for consumers in the eating imagery condition.

Supporting Hypothesis 1, eur the results revealed a significant interaction between the mental imagery condition and the size of the food portion on the expected pleasure of eating it. Participants in the eating imagery condition rated the smaller portion of food as being more appetizing that those in the control imagery condition. However, mental imagery did not lead to a more positive evaluation of the more positively-valenced option, and to a less positive evaluation of the less positively-valenced option. Indeed, no difference in expected pleasure evaluation between the two options was found.

Interestingly, a significant interaction of our the independent variables on calorie perception was observed. Participants in the imagined eating condition perceived the smaller food portion to be more calorific than those participants in the control imagery condition, and 
interestingly more calorific than the larger food portion. One interpretation for this result could be that imagining the sensory experience of eating facilitated the estimation of calorie content that is generally underestimated (Wansink \& Chandon, 2006). It has beenA previous study has shown that the fact that theestimation of calorie content is easier for smaller food portions than for larger food portions (Wansink \& Chandon, 2006). This resultcan perhaps help to explain why imagining the experience of eating has only had an effect on the calorie estimation of the smaller food portion.

People in the eating imagery condition evaluated the smaller food portion as more calorific and more appetizing than those in the control imagery condition. Brunstrom and Shakeshaft (2009) have shown that consumers generally consider liked food and food that is more satiating to be more rewarding. They also prefer smaller portions of foods that are regarded as more rewarding. For this reason, it can be supposed that imagining the sensory experience of eating the portion of food could increase the reward value of the smaller portion but not the larger portion.

Note, neither the level of hunger, nor the purchase intent were affected by ourthe independent variables. Thus, eur the results do not support Hypotheses 2 and 3. One explanation for these null results might be that the larger food portion was not large enough to produce satiety by eating imagery (Larson et al., 2014; Morewedge et al., 2010). These results also suggest that asking people explicitly to imagine the sensory experience of eating the food does not affect their motivation to get the food, contrary to the implicit simulation of eating. Indeed, Study 1 revealed a main effect of eating simulations on the level of hunger and purchase intent. Thus, we can suppose that asking people to imagine the sensory experience of eating the food could simply helped them to better consider the food portion size.

\section{General discussion}




\subsection{Summary}

This article offers several contributions to research on the size of food portions, and provides managerial insight related to the efficacy of asking people to imagine the eating experience in health prevention and food marketing campaigns. In particular, this research constitutes the first empirical evidence to show that it would be possible to reduce the portion size effect by imagining the experience of eating a food portionimagining the experience of eating a food portion would reduce the portion size effect. The results of Study 1 revealed that consumers with higher eating simulation scores found the food to be more appetizing, and expressed greater purchase intentions than those consumers with lower scores. This result confirms that when people spontaneously simulate the consumption of food; it that reflects a greater motivation to obtain the food (Jeannerod, 2001; Schlosser, 2003). The results of Study 1 also demonstrate that the spontaneous simulation of eating a large amount of food can result in reduced hunger (Larson et al., 2014), as people indicate feeling less hunger after seeing the very large portion, than after seeing the smaller food portions.

MoreoverFurthermore, people with higher eating simulation scores select less food than those consumers with lower scores in the very large portion. The percent of food selected by those participants was also smaller in the very large portion than in the medium food portion.

However, they selected more food in this portion than in the medium portion, thus suggesting that spontaneous simulation of eating experiences reduce the portion size effect, but does not eliminate it entirely.

The results of Study 2 indicate that it is pessible to reduce the portion size effect can be reduced significantly simply by asking consumers to imagine the sensory experience of eating the entire portion of food. Contrary to consumers in the control imagery condition, those in the eating imagery condition did not select significantly more food in the larger portion than in the smaller portion. Interestingly, participants in the eating imagery condition 
evaluated the smaller food portion as more appetizing and more calorific than those in the control imagery condition. This result may indicate that imagining the sensory experience of eating not only increases the expected pleasure of smaller food portions, but might also increase expected satiety (i.e., by increasing the perception of the calorie content), that enhances the rewarding value of the smaller food portion (Brunstrom \& Shakeshaft, 2009). For this reason, it might be interesting to include expected satiety might be included in future research on this topic (Forde, Almiron-Roig, \& Brunstrom, 2015). Moreover $\underline{\text { In addition, }}$ people in the eating imagery condition rated the smaller portion of food as being more calorific than the larger food portion. Since, the calorie content of a food portion, especially a large portion, is typically underestimated, it can be supposed that-imagining the sensory experience of eating might helps people to estimate the caloric content more easily for the smaller food portion (Wansink \& Chandon, 2006).

An alternative explanation for the reduced amount of food selected from the larger portion would be that the act of imagining eating the whole portion makes people realize that the food portion that has been presented would be too much, so that the portion size effect disappears. Thus, a reduction in purchase intentions might have been expected for the larger food portion. However, eur-the results do not support this explanation, since we did not find an effect of the food portion size on purchase intentions was not found. Since, participants in the eating imagery condition have the same purchase intentions and select the same amount of food from both portions, we may well imagine that asking people to imagine the sensory experience of eating-could simply help_ed them to better consider the food portion size without increasing their motivation to acquire the food. For this reason, it could be interestingnew studies would be conducted to test whether imagining the sensory experience of eating may help the consumer to select more "optimal" portions at the supermarket or at the restaurant. 
It can be argued that the The two studies reported here have implications both for public policy and for the food industry. Portion size significantly influences energy intake (Rolls et al., 2002; Wansink et al., 2005; Zlatevska et al., 2014). By asking consumers to imagine the sensory experience of eating the entire portion of food, the portion size effect can be reduced; a tangible application of this would be in the form of a health campaign to promote the mental imagery of eating the food portion (Petit et al., 2016a, b). The food industry could also follow this strategy, with, for example, Cornil and Chandon (in press) demonstrating that those consumers asked to imagine the multisensory experience of eating expect more pleasure, and are willing to pay more for smaller food portions than for larger food portions. Likewise, in the present study, asking people to imagine the sensory experience of eating increased the reward value of the smaller food portion. Thus, by promoting the sensory experience of eating a smaller food portion, food marketers might reduce their economic costs associated with smaller food portions.

\subsection{Limitations and future research}

The main limitation of the present research is to have only tested the effect of simulation of eating on the serving of food, but not on consumption itself, which could well reduce the impact of satiety on consumption regulation. That is, even if people with lower eating simulation scores report being less hungry after seeing the very large portion than the smaller portions, they continue to select more from this larger portion, since they did not have to eat the food. However, given that people with higher eating simulation scores are able to reduce the percent of food select inside the larger food portions, just by viewing the portion on the screen, we can expect that this result would be enhanced by a real consumption.

However, it is still encouraging to see these results are still encouraging, as they suggest that it would be possible to modify people's eating intentions would be modified.

In contrast, Marchiori and Papies (2014) were unable to reduce the portion size effect 
by having their consumers perform a mindfulness exercise (i.e., which increased the attention of consumers to their body sensations). In their study, different portions of food were placed near the participant during the exercise, and the amount of food eaten by participants was recorded. It is possible that they did not find a positive effect because their exercise was not focused on the sensory experience that appears to make the smaller food portion more rewarding. Future studies may extend eur the results and assess the effect of eating imagery on food portion consumption.

Another important limitation of the present research is not having asked the participants to specify what kind of simulation of eating experiences they had when they saw the food portion (Study 1). As eating simulations can refer to sensory (e.g., "crunchy"; “sweet", "cold”), situational (e.g. "evening, "sofa", "party”), and hedonic (“tasty", “delicious”, “disgusting”) features (Papies, 2013), and since we asked the participants were asked to imagine the sensory experience of eating in Study 2, future research should test which eating simulation features are more likely to reduce the portion size effect.

In Study 2, the portion size effect was reduced significantly by asking people to imagine the eating experience, but we did not find a positive effect of this eating imagery on purchase intent was not found. This result suggests that explicitly asking people to imagine the experiences associated with eating is less motivating than when this experience arises spontaneously. Future studies should therefore be conducted in order to test how food marketing and health prevention campaigns can fuel spontaneous imagery of eating experiences (Petit et al., 2016a). For instance, we can imagine promoting short texts in a food portion sized packaging (e.g., "Imagine the pleasure inside") could help to enhance purchase intent for smaller food portions.

To conclude, here we repert that mental simulation of a consumption experience can act to reduce the total quantity of a portion of food a person wishes to consume. The obvious 
next step, which arewill be exploreding, is to see if such intentions translate into real calories consumed. 


\section{REFERENCES}

Anderson, C. A. (1983). Imagination and expectation: The effect of imagining behavioral scripts on personal influences. Journal of Personality and Social Psychology, 45(2), 293-305.

Barsalou, L. W. (2008). Grounded cognition. Annual Review of Psychology, 59, 617-645.

Barsalou, L. W., Simmons, W. K., Barbey, A. K., \& Wilson, C. D. (2003). Grounding conceptual knowledge in modality-specific systems. Trends in Cognitive Sciences, 7(2), 84-91.

Birch, L. L., Engell, D., \& Rolls, B. J. (2000). Serving portion size influences 5-year-old but not 3-year-old children's food intakes. Journal of the American Dietetic Association, 100(2), 232-234.

Birch, L. L., McPhee, L., Shoba, B. C., Steinberg, L., \& Krehbiel, R. (1987). “Clean up your plate": Effects of child feeding practices on the conditioning of meal size. Learning and Motivation, 18(3), 301-317.

Brunstrom, J. M., \& Shakeshaft, N. G. (2009). Measuring affective (liking) and non-affective (expected satiety) determinants of portion size and food reward. Appetite, 52(1), 108114.

Chandon, P., \& Wansink, B. (2012). Does food marketing need to make us fat? A review and solutions. Nutrition Reviews, 70(10), 571-593.

Chen, J., Papies, E. K., \& Barsalou, L. W. (in press). A core eating network and its modulations underlie diverse eating phenomena. Brain and Cognition.

Cornil, Y., \& Chandon, P. (in press). Pleasure as a substitute for size: How multisensory imagery can make people happier with smaller food portions. Journal of Marketing Research. 
de Graaf, C. (2012). Texture and satiation: The role of oro-sensory exposure time. Physiology \& Behavior, 107(4), 496-501.

Elder, R. S., \& Krishna, A. (2012). The "visual depiction effect" in advertising: Facilitating embodied mental simulation through product orientation. Journal of Consumer Research, 38(6), 988-1003.

Forde, C. G., Almiron-Roig, E., \& Brunstrom, J. M. (2015). Expected satiety: Application to weight management and understanding energy selection in humans. Current Obesity Reports, 4(1), 131-140.

Galak, J., Redden, J. P., \& Kruger, J. (2009). Variety amnesia: Recalling past variety can accelerate recovery from satiation. Journal of Consumer Research, 36(4), 575-584.

Garbinsky, E. N., Morewedge, C. K., \& Shiv, B. (2014). Interference of the end: Why recency bias in memory determines when a food is consumed again. Psychological Science, 29(7), 1466-1474.

Geier, A. B., Rozin, P., \& Doros, G. (2006). Unit bias a new heuristic that helps explain the effect of portion size on food intake. Psychological Science, 17(6), 521-525.

Gregory, W. L., Cialdini, R. B., \& Carpenter, K. M. (1982). Self-relevant scenarios as mediators of likelihood estimates and compliance: Does imagining make it so? Journal of Personality and Social Psychology, 43(1), 89-99.

Herman, C. P., \& Polivy, J. (1983). A boundary model for the regulation of eating. Psychiatric Annals, 13(12), 918-927.

Iacobucci, D., Posavac, S. S., Kardes, F. R., Schneider, M. J., \& Popovich, D. L. (2015). Toward a more nuanced understanding of the statistical properties of a median split. Journal of Consumer Psychology, 25(4), 652-665.

Jeannerod, M. (2001). Neural simulation of action: A unifying mechanism for motor cognition. NeuroImage, 14(1), S103-S109. 
Kral, T. V. E., Roe, L. S., \& Rolls, B. J. (2004). Combined effects of energy density and portion size on energy intake in women. American Journal of Clinical Nutrition, 79, 962-968.

Krishna, A., \& Schwarz, N. (2014). Sensory marketing, embodiment, and grounded cognition: A review and introduction. Journal of Consumer Psychology, 24(2), 159168.

Larson, J., Redden, J. P., \& Elder, R. (2014). Satiation from sensory simulation: Evaluating foods decreases enjoyment of similar foods. Journal of Consumer Psychology, 24(2), 188-194.

Marchiori, D., \& Papies, E. K. (2014). A brief mindfulness intervention reduces unhealthy eating when hungry, but not the portion size effect. Appetite, 75, 40-45.

Morewedge, C. K., Huh, Y. E., \& Vosgerau, J. (2010). Thought for food: Imagined consumption reduces actual consumption. Science, 330(6010), 1530-1533.

Papies, E. K. (2013). Tempting food words activate eating simulations. Frontiers in Psychology, 4:838.

Petit, O., Basso, F., Merunka, D., Spence, C., Cheok, A. D., \& Oullier, O. (2016a). Pleasure and the control of food intake: An embodied cognition approach to consumer self regulation. Psychology \& Marketing, 33(8), 608-619.

Petit, O., Merunka, D., Anton, J. L., Nazarian, B., Spence, C., Cheok, A. D., \& Oullier, O. (2016b). Health and pleasure in consumers' dietary food choices: Individual differences in the brain's value system. PLOS ONE, 11(7):e0156333.

Ramaekers, M. G., Luning, P. A., Ruijschop, R. M., Lakemond, C. M., Bult, J. H., Gort, G., \& van Boekel, M. A. (2014). Aroma exposure time and aroma concentration in relation to satiation. British Journal of Nutrition, 111(3), 554-562. 
Redden, J. P., \& Haws, K. L. (2013). Healthy satiation: The role of decreasing desire in effective self-control. Journal of Consumer Research, 39(5), 1100-1114.

Rolls, B. J., Morris, E. L., \& Roe, L. S. (2002). Portion size of food affects energy intake in normal-weight and overweight men and women. American Journal of Clinical Nutrition, 76(6), 1207-1213.

Schlosser, A. E. (2003). Experiencing products in the virtual world: The role of goal and imagery in influencing attitudes versus purchase intentions. Journal of Consumer Research, 30(2), 184-198.

Simmons, W. K., Martin, A., \& Barsalou, L. W. (2005). Pictures of appetizing foods activate gustatory cortices for taste and reward. Cerebral Cortex, 15(10), 1602-1608.

Spence, C., Okajima, K., Cheok, A. D., Petit, O., \& Michel, C. (in press). Eating with our eyes: From visual hunger to digital satiation. Brain and Cognition.

Toepel, U., Bielser, M. L., Forde, C., Martin, N., Voirin, A., le Coutre, J., Murray, M. M., \& Hudry, J. (2015). Brain dynamics of meal size selection in humans. NeuroImage, $113(1), 133-142$.

Van der Laan, L. N., De Ridder, D. T. D., Viergever, M. A., \& Smeets, P. A. (2011). The first taste is always with the eyes: A meta-analysis on the neural correlates of processing visual food cues. NeuroImage, 55(1), 296-303.

Vermeer, W. M., Alting, E., Steenhuis, I. H., \& Seidell, J. C. (2010). Value for money or making the healthy choice: the impact of proportional pricing on consumers' portion size choices. The European Journal of Public Health, 20(1), 65-69.

Versluis, I., \& Papies, E. K. (2016). The role of social norms in the portion size effect: Reducing normative relevance reduces the effect of portion size on consumption decisions. Frontiers in Psychology, 7:756. 
Wansink, B. (2004). Environmental factors that increase the food intake and consumption volume of unknowing consumers. Annual Review of Nutrition, 24, 455-479.

Wansink, B., \& Chandon, P. (2006). Meal size, not body size, explains errors in estimating the calorie content of meals. Annals of Internal Medicine, 145(5), 326-332.

Wansink, B., \& Kim, J. (2005). Bad popcorn in big buckets: Portion size can influence intake as much as taste. Journal of Nutrition Education and Behavior, 37(5), 242-245.

Wansink, B., Painter, J. E., \& North, J. (2005). Bottomless bowls: Why visual cues of portion size may influence intake. Obesity Research, 13(1), 93-100.

Wansink, B., \& Van Ittersum, K. (2007). Portion size me: Downsizing our consumption norms. Journal of the American Dietetic Association, 107(7), 1103-1106.

Weijzen, P. L., Smeets, P. A., \& de Graaf, C. (2009). Sip size of orangeade: Effects on intake and sensory-specific satiation. British Journal of Nutrition, 102(7), 1091-1097.

Woods, A. T., Velasco, C., Levitan, C. A., Wan, X., \& Spence, C. (2015). Conducting perception research over the internet: A tutorial review. PeerJ, 3:e1058.

Zlatevska, N., Dubelaar, C., \& Holden, S. S. (2014). Sizing up the effect of portion size on consumption: A meta-analytic review. Journal of Marketing, 78(3), 140-154. 


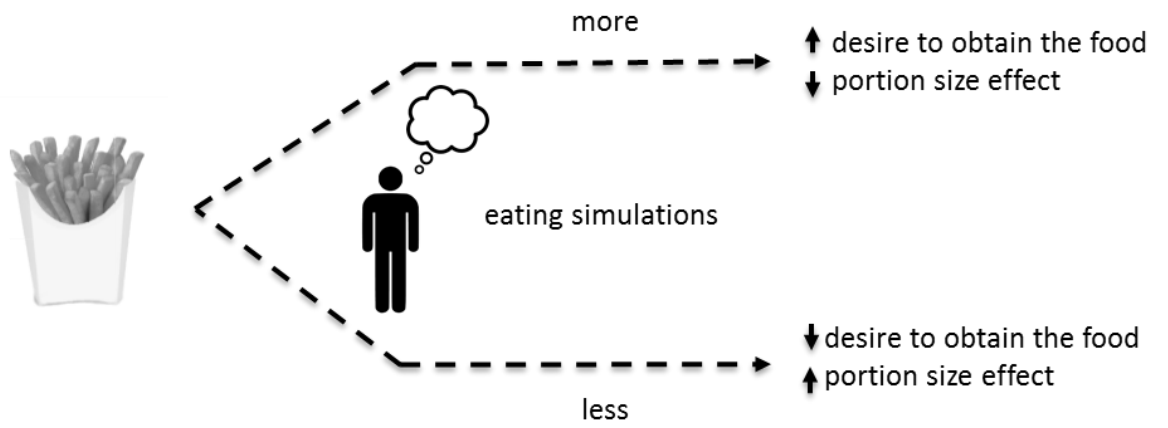

Fig. 1. Schematic representation of the expected differences between people experiencing more eating simulations vs. less eating simulations on the desire to obtain the food, and portion size effect, when they see a portion of food. 
Table 1.

Study 1: Descriptive statistics

\begin{tabular}{llllllll}
\hline $\begin{array}{l}\text { Portion } \\
\text { size }\end{array}$ & $\begin{array}{l}\text { Eating } \\
\text { simulations }\end{array}$ & $\begin{array}{l}\text { Caloric content } \\
\text { (calories) }\end{array}$ & $\begin{array}{l}\text { Expected } \\
\text { pleasure }\end{array}$ & $\begin{array}{l}\text { Purchase } \\
\text { intent }\end{array}$ & Hunger & $\begin{array}{l}\text { Food selected } \\
\text { (number) }\end{array}$ & (percent) \\
\hline Medium & High $N=24$ & $20.73(9.48)^{\mathrm{a}}$ & $4.25(1.57)$ & $3.54(1.87)$ & $3.42(1.61)$ & $7.00(6.41)$ & $39.38(37.54)$ \\
& Low $N=21$ & $32.86(20.46)$ & $5.86(1.20)$ & $4.81(1.83)$ & $5.00(1.41)$ & $9.86(7.27)$ & $57.98(42.76)$ \\
& Total $N=45$ & $26.39(16.57)$ & $5.00(1.61)$ & $4.13(1.94)$ & $4.16(1.71)$ & $8.33(6.90)$ & $48.06(40.69)$ \\
Large & High $N=25$ & $21.07(10.90)$ & $4.16(1.52)$ & $3.72(1.79)$ & $3.80(1.73)$ & $12.68(8.92)$ & $48.77(34.29)$ \\
& Low $N=25$ & $28.26(16.52)$ & $5.76(1.09)$ & $5.57(1.33)$ & $4.20(1.61)$ & $14.32(8.16)$ & $55.08(31.38)$ \\
& Total $N=50$ & $24.67(14.32)$ & $4.96(1.54)$ & $4.64(1.82)$ & $4.00(1.67)$ & $13.50(8.50)$ & $51.92(32.68)$ \\
Very large & High $N=22$ & $28.43(16.20)$ & $3.59(1.56)$ & $3.18(1.76)$ & $2.82(1.47)$ & $29.06(12.21)$ & $56.95(23.94)$ \\
& Low $N=25$ & $34.44(19.28)$ & $5.44(1.42)$ & $4.64(1.60)$ & $3.44(1.71)$ & $18.60(13.86)$ & $36.47(27.18)$ \\
Total & Total $N=47$ & $31.63(17.97)$ & $4.58(1.74)$ & $3.96(1.82)$ & $3.15(1.62)$ & $23.49(14.00)$ & $46.06(27.46)$ \\
& High $N=71$ & $23.26(12.70)$ & $4.01(1.55)$ & $3.50(1.80)$ & $3.37(1.64)$ & $15.83(13.06)$ & $48.13(33.00)$ \\
& Low $N=71$ & $31.80(18.65)$ & $5.68(1.24)$ & $5.01(1.62)$ & $4.17(1.69)$ & $14.51(10.78)$ & $49.38(34.74)$ \\
& Total $N=142$ & $27.52(16.47)$ & $4.85(1.63)$ & $4.25(1.87)$ & $3.77(1.71)$ & $15.17(11.95)$ & $48.76(33.76)$ \\
\hline
\end{tabular}

${ }^{a}$ Numbers in parentheses represent standard errors. 


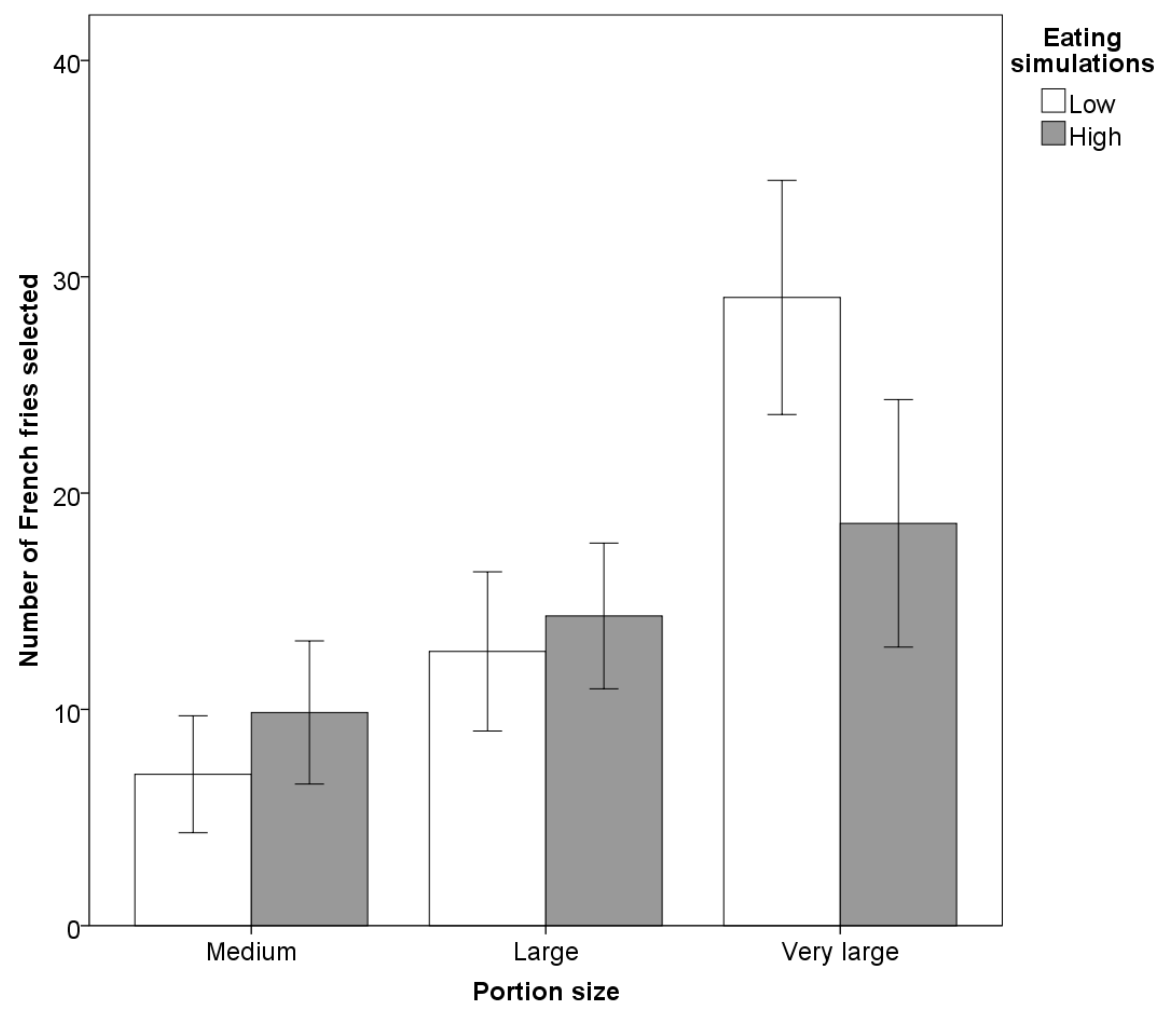

Fig. 2. Number of French fries selected from the portion by consumers with lower versus

higher eating simulation scores in medium, large, and very large portions, in Experiment 1.

Commented [CS21]: don't you call it study in main text

Error bars represent the $95 \%$ confidence intervals. 
Table 2.

Study 2: Descriptive statistics

\begin{tabular}{llllllll}
\hline $\begin{array}{l}\text { Portion } \\
\text { size }\end{array}$ & Mental imagery & $\begin{array}{l}\text { Caloric content } \\
\text { (calories) }\end{array}$ & $\begin{array}{l}\text { Expected } \\
\text { pleasure }\end{array}$ & $\begin{array}{l}\text { Purchase } \\
\text { intent }\end{array}$ & Hunger & $\begin{array}{l}\text { Food selected } \\
\text { (number) }\end{array}$ & (percent) \\
\hline Smaller & Control $N=49$ & $26.59(17.52)$ & $4.78(1.71)$ & $3.71(2.04)$ & $4.04(1.58)$ & $7.06(4.71)$ & $47.08(31.42)$ \\
& Eating $N=50$ & $37.10(15.59)$ & $5.60(1.44)$ & $4.58(1.63)$ & $4.00(1.70)$ & $8.92(4.96)$ & $59.47(33.07)$ \\
& Total $N=99$ & $31.90(17.31)$ & $5.19(1.63)$ & $4.15(1.89)$ & $4.02(1.64)$ & $8.00(4.91)$ & $53.33(32.70)$ \\
Larger & Control $N=50$ & $28.23(19.32)$ & $5.28(1.81)$ & $4.54(2.12)$ & $4.26(1.76)$ & $11.60(6.87)$ & $48.33(28.61)$ \\
& Eating $N=48$ & $27.51(17.48)$ & $5.13(1.63)$ & $4.40(2.01)$ & $4.04(1.56)$ & $9.69(6.45)$ & $40.57(26.74)$ \\
& Total $N=98$ & $27.88(18.35)$ & $5.20(1.72)$ & $4.47(2.06)$ & $4.15(1.66)$ & $10.66(6.70)$ & $44.53(27.84)$ \\
Total & Control $N=99$ & $27.42(18.38)$ & $5.03(1.77)$ & $4.13(2.11)$ & $4.15(1.67)$ & $9.35(6.30)$ & $47.71(29.89)$ \\
& Eating $N=98$ & $32.40(17.15)$ & $5.37(1.55)$ & $4.49(1.82)$ & $4.02(1.62)$ & $9.30(5.72)$ & $50.21(31.45)$ \\
& Total $N=197$ & $29.90(17.91)$ & $5.20(1.67)$ & $4.31(1.97)$ & $4.09(1.64)$ & $9.33(6.00)$ & $48.96(30.62)$ \\
\hline
\end{tabular}

${ }^{\mathrm{a}}$ Numbers in parentheses represent standard errors. 


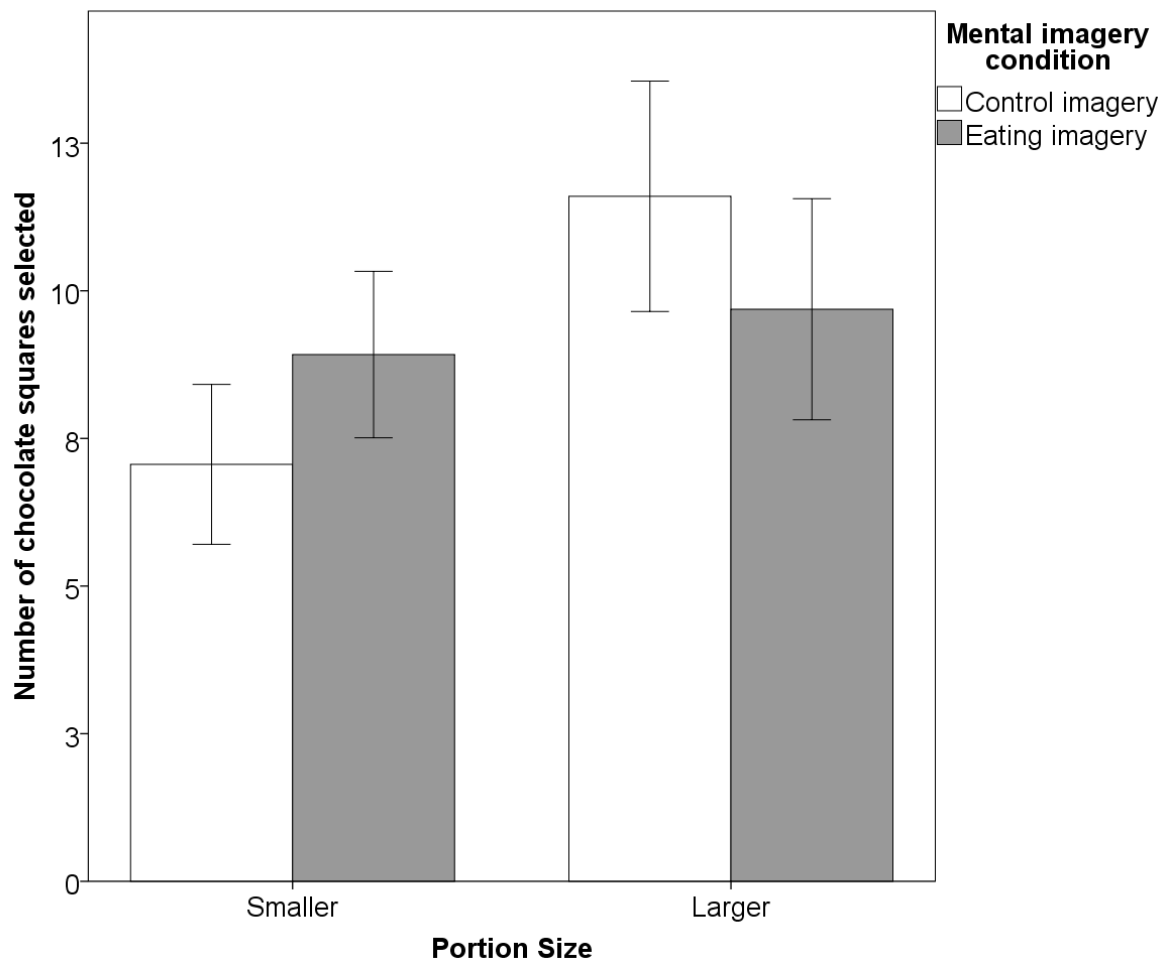

Fig. 3. Number of chocolate's squares selected in the smaller and larger chocolate bar sizes, by consumers in the control and in the eating imagery conditions, in Experiment 2. Error bars represent the $95 \%$ confidence intervals. 


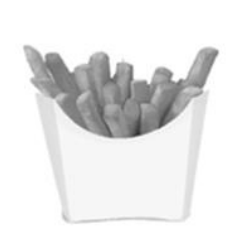

Medium

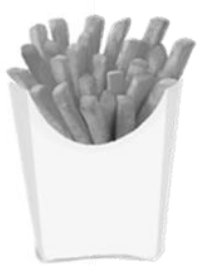

Large

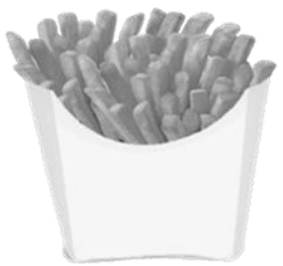

Very large

Appendix A.1. French fries portion sizes (medium, large, and very large).

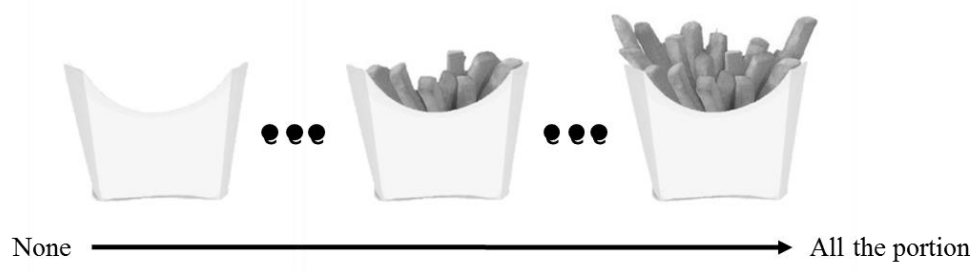

Appendix A.2. Medium version of the French fries scale.
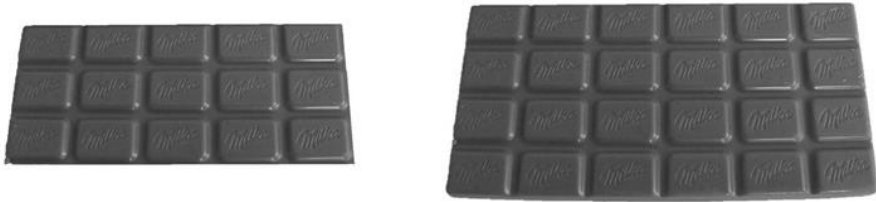

Appendix B. Chocolate bar size (from left to right: smaller and larger portions). 